\title{
Using zebrafish to model haemorrhagic stroke
}

Paul Kasher, Siobhan Crilly, Alex Njegic, Elisavet Fotiou, Sarah Laurie, James Cave, Adrian-Parry Jones, Stuart Allan The University of Manchester, UK

paul.kasher@manchester.ac.uk

\section{The zebrafish model system}

Zebrafish are vertebrates with a well conserved vascular system that is highly appropriate for studying intracerebral haemorrhage $(\mathrm{ICH})$ pre-clinically.

Highly amenable to genetic manipulation and high-throughput drug screening.

Larval transparency + transgenic fluorescent reporter lines $=$ powerful tool for studying cellular biology in real time, in intact animals.

Spontaneous blood vessel rupture can be induced within the brain using chemical or genetic techniques - i.e. non-invasive.

Using live imaging, we can make observations in zebrafish larvae that are not possible in rodent models of $\mathrm{ICH}$.

\section{ICH in zebrafish larvae induces a quantifiable brain injury}

Cell death was assessed using a transgenic fluorescent reporter zebrafish line that expresses a yellow/green fluorescent protein in dying cells (annexinV:ubiq:YFP).

Significantly increased rates of cell death was observed in the brains of haemorrhaged $(\mathrm{ICH}+)$ larvae in comparison to nonhaemorrhage siblings ( $\mathrm{ICH}-)$.

Cell death 'clusters' were always situated adjacent to the haemorrhage site (asterisks) from 3dpf.
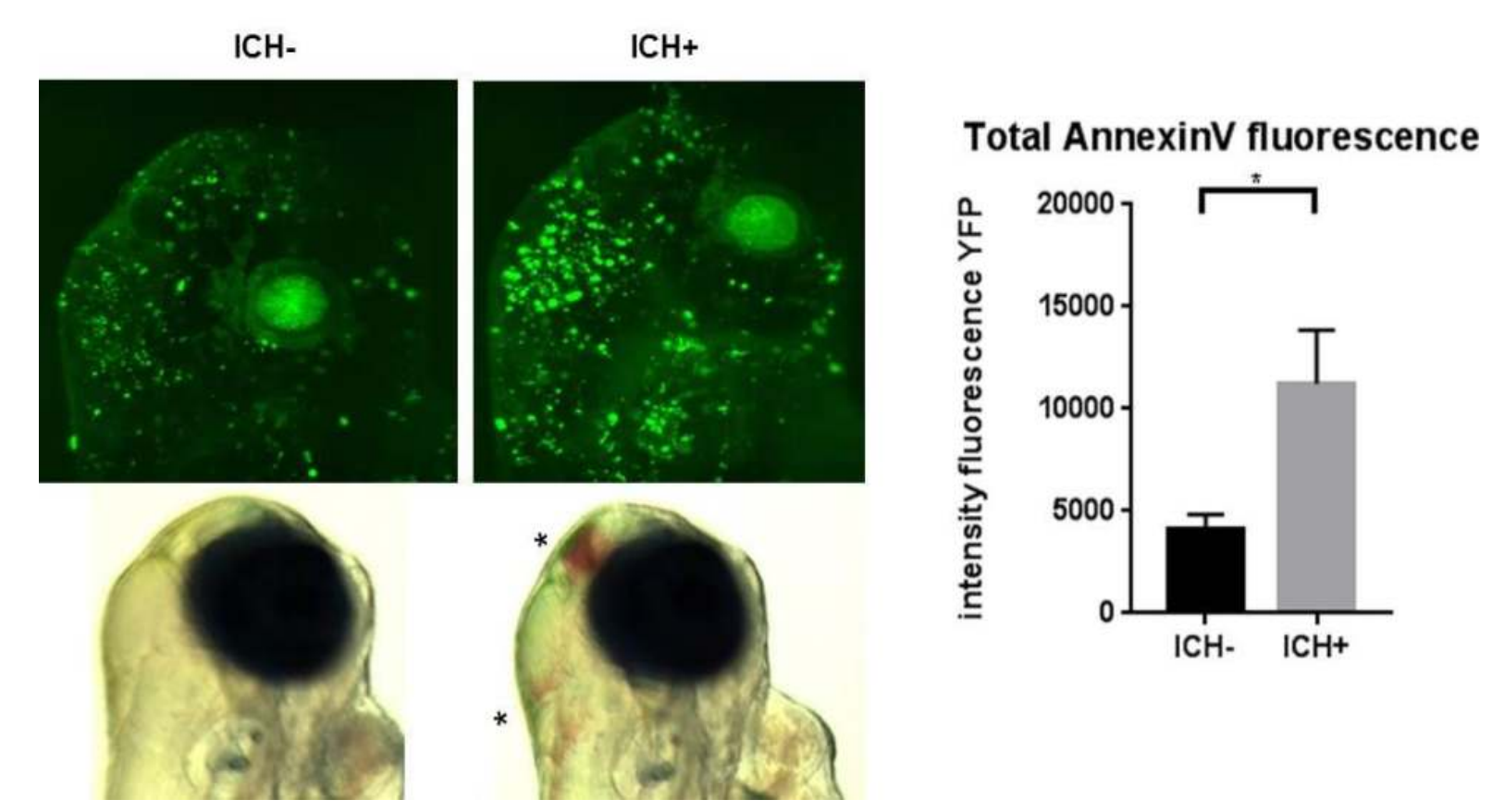

\section{ICH in zebraffish larvae induces an innate immune response}

Post- $\mathrm{ICH}$ neuroinflammation was assessed using transgenic fluorescent reporter zebrafish lines for neutrophils (mpo:GFP) and macrophages (mpeg 1:mCherry).

At 3dpf, a significant increase in the number of macrophages, but not neutrophils, recruited to the brain was observed in $\mathrm{ICH}+$ larvae $(\mathrm{A})$.

Significantly increased numbers of activated (amoeboid) macrophages were also observed in $\mathrm{ICH}+$ larval brains $(B, C)$.

Expression of the pro-inflammatory cytokine gene, interleukin$1 \beta$, was significantly increased following $\mathrm{ICH}(\mathrm{D})$.

A

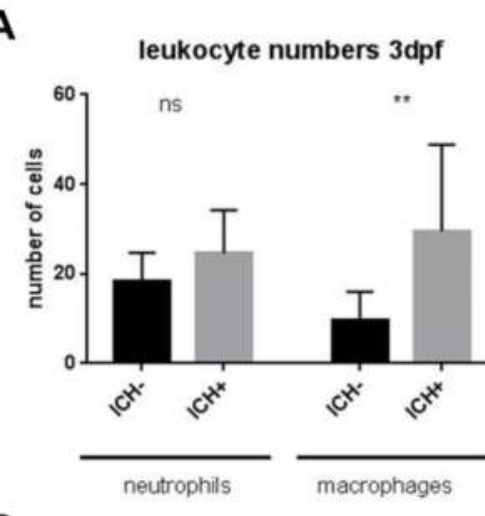

C

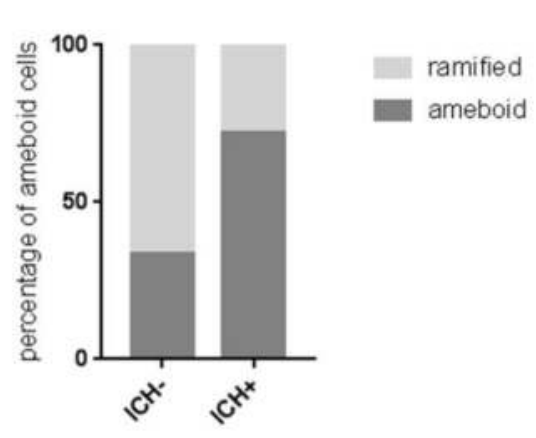

B ICH-

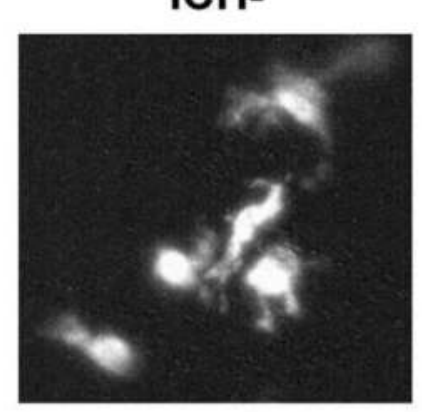

D

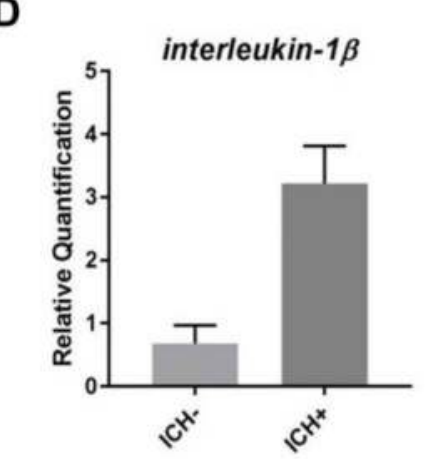

\section{Non-invasive induction of spontaneous ICH}

Brain-specific bleeding induced from 2 days post-fertilisation (dpf) in zebrafish larvae

Haemorrhages (arrows) were assessed in live animals using a double transgenic reporter zebrafish line that express a green fluorescent protein in blood vessels (fli1:GFP) and a red fluorescent protein in erythrocytes (gata:DSRed).

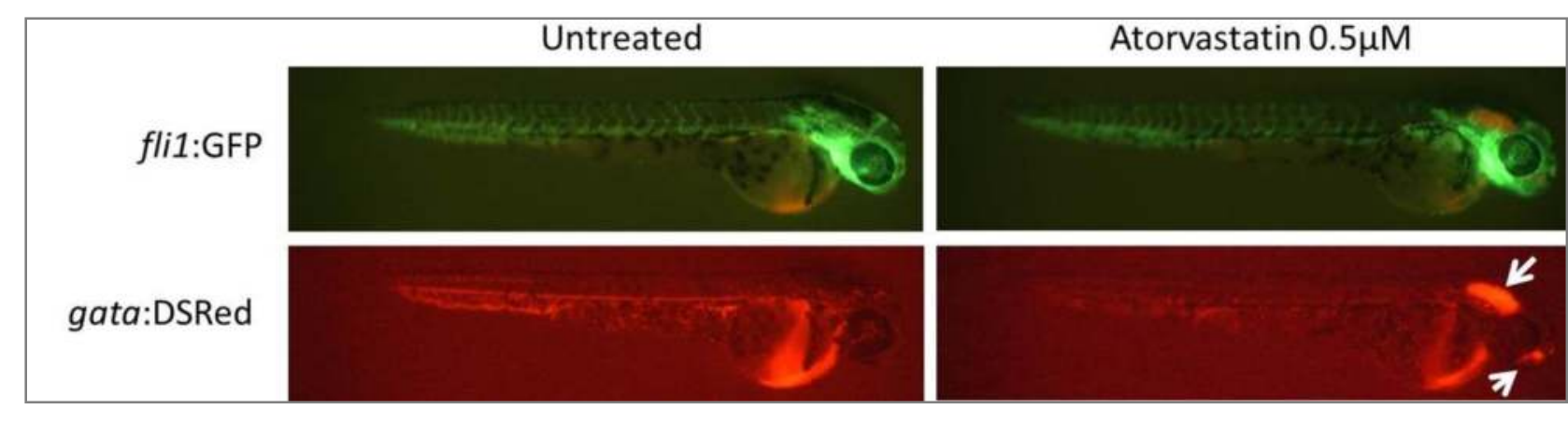

\section{ICH in zebrafish larvae induces a quantifiable locomotor deficit}

Swimming ability was assessed using an automated tracking system (Daniovision) to quantify locomotor function following $\mathrm{ICH}$ induced brain injury.

$\mathrm{ICH}+$ and $\mathrm{ICH}$ - larval siblings were tested at 3, 4 and 5dpf.

Significant reductions in total distance moved was recorded following $\mathrm{ICH}$ at all three time points.
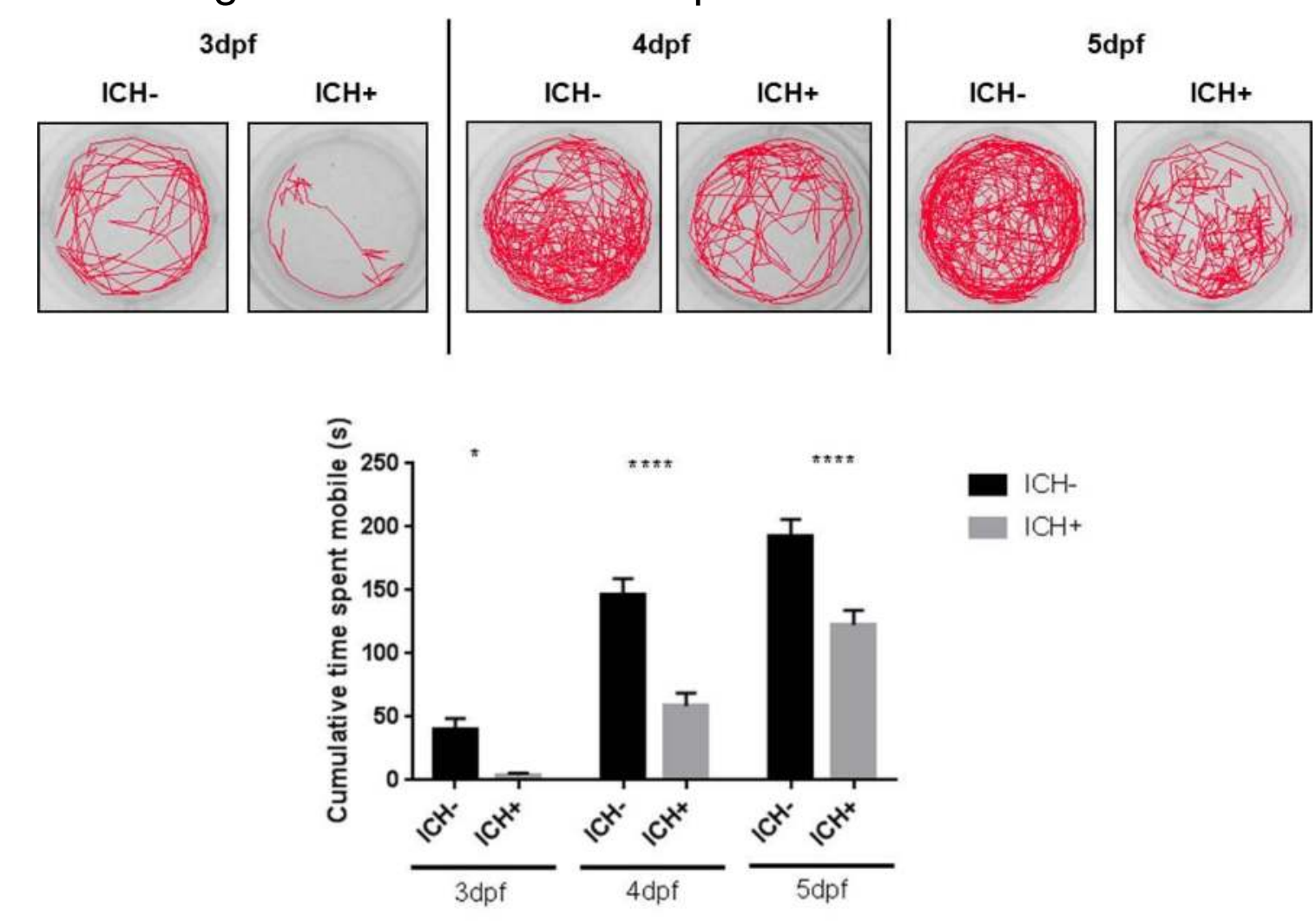

6. Summary

Zebrafish offer a rapid and accessible disease model system for pre-clinical ICH research.

Unlike rodent models, spontaneous $\mathrm{ICH}$ can be induced in zebrafish larvae using non-invasive procedures.

In zebrafish, bleeding in the brain causes quantifiable and reproducible brain injury, locomotor and neuroinflammatory phenotypes.

Importantly, these phenotypes recapitulate key pathological features of the human condition, thereby validating the use of zebrafish as an alternative pre-clinical system for $\mathrm{ICH}$ research.

This model can be used as a new platform for improving our understanding of basic ICH pathophysiology....

....and for future translational drug discovery studies.

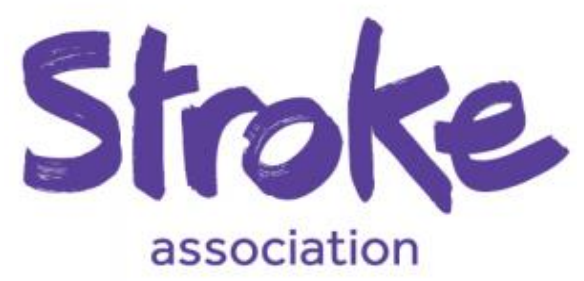

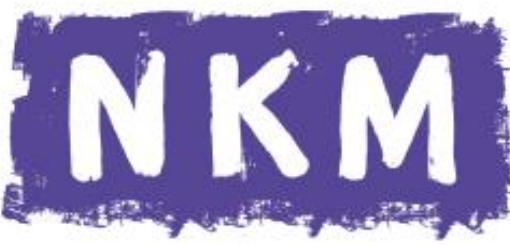

THE NATALIE KATE MOSS TRUST

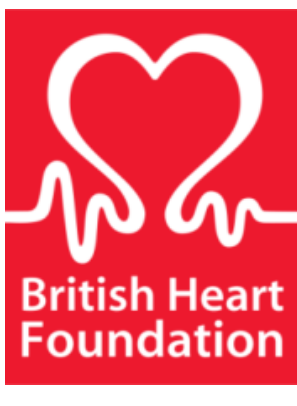

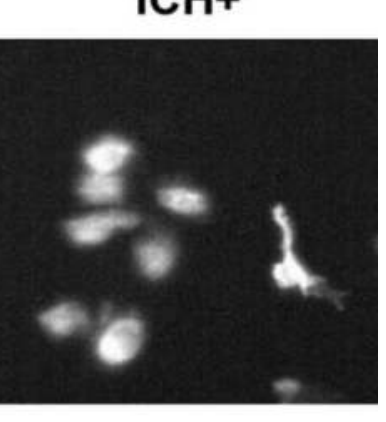

